OPEN ACCESS

Edited by:

Juan B. Barroso,

University of Jaén, Spain

Reviewed by:

Oscar Lorenzo

University of Salamanca, Spain

Renaud Brouquisse,

INRA Centres Provence-Alpes-Côte

d'Azur, France

*Correspondence:

Lorenzo Lamattina

lolama@mdp.edu.ar

${ }^{\dagger}$ These authors have contributed equally to this work

Specialty section: This article was submitted to Plant Physiology,

a section of the journal Frontiers in Plant Science

Received: 12 September 2019 Accepted: 03 March 2020

Published: 20 March 2020

Citation:

Nejamkin A, Foresi N, Mayta ML,

Lodeyro AF, Del Castello F, Correa-Aragunde N, Carrillo N and Lamattina L (2020) Nitrogen Depletion Blocks Growth Stimulation Driven by the Expression of Nitric Oxide

Synthase in Tobacco.

Front. Plant Sci. 11:312.

doi: 10.3389/fpls.2020.00312

\section{Nitrogen Depletion Blocks Growth Stimulation Driven by the Expression of Nitric Oxide Synthase in Tobacco}

\author{
Andrés Nejamkin ${ }^{1 \dagger}$, Noelia Foresi1t, Martín L. Mayta ${ }^{2}$, Anabella F. Lodeyro², \\ Fiorella Del Castello ${ }^{1}$, Natalia Correa-Aragunde ${ }^{1}$, Néstor Carrillo ${ }^{2}$ and \\ Lorenzo Lamattina ${ }^{1 *}$ \\ ${ }^{1}$ Instituto de Investigaciones Biológicas, Facultad de Ciencias Exactas y Naturales, Universidad Nacional de Mar del Plata, \\ Mar del Plata, Argentina, ${ }^{2}$ Instituto de Biología Molecular y Celular de Rosario (IBR-CONICET), Facultad de Ciencias \\ Bioquímicas y Farmacéuticas, Universidad Nacional de Rosario, Rosario, Argentina
}

Nitric oxide (NO) is a messenger molecule widespread studied in plant physiology. Latter evidence supports the lack of a NO-producing system involving a NO synthase (NOS) activity in higher plants. However, a NOS gene from the unicellular marine alga Ostreococcus tauri (OtNOS) was characterized in recent years. OtNOS is a genuine NOS, with similar spectroscopic fingerprints to mammalian NOSs and high NO producing capacity. We are interested in investigating whether OtNOS activity alters nitrogen metabolism and nitrogen availability, thus improving growth promotion conditions in tobacco. Tobacco plants were transformed with OtNOS under the constitutive CaMV 35S promoter. Transgenic tobacco plants expressing OtNOS accumulated higher NO levels compared to siblings transformed with the empty vector, and displayed accelerated growth in different media containing sufficient nitrogen availability. Under conditions of nitrogen scarcity, the growth promoting effect of the OtNOS expression is diluted in terms of total leaf area, protein content and seed production. It is proposed that OtNOS might possess a plant growth promoting effect through facilitating $\mathrm{N}$ remobilization and nitrate assimilation with potential to improve crop plants performance.

Keywords: nitric oxide, nitric oxide synthase, Nicotiana tabacum, nitrogen, plant growth, seed production

\section{INTRODUCTION}

Crop productivity depends on strong nitrogen $(\mathrm{N})$ fertilization, though plants use only $50 \%$ of the supplied nitrogen. For most plant species, NUE is defined by the plant capacity to extract inorganic nitrogen $(\mathrm{N})$ from the soil, assimilate nitrate and ammonium, translocate, remobilize and recycle of organic $\mathrm{N}$ forms during the life cycle (Krstić and Sarić, 1983). Metabolic processes based on protein synthesis and $\mathrm{N}$-containing biomolecules are critical for plant vegetative and reproductive growth and yield, and dependent on the adequate N supply (Sinclair and Rufty, 2012). Thus, improving NUE is a big challenge for plant biotechnology (Masclaux-Daubresse et al., 2010).

Both field and laboratory researches have demonstrated that increasing the supply of $\mathrm{N}$ fertilizers enhances growth and photosynthesis. The sensitivity for $\mathrm{N}$ fertilization is species specific and

Abbreviations: NO, nitric oxide; NUE, nitrogen use efficiency; OtNOS, nitric oxide synthase from Ostreococcus tauri. 
central for agriculture. Nitrogen scarcity results in a reduced leaf area and leaf energy production due to a reduced light interception for photosynthesis (Huber et al., 1989; Tóth et al., 2002; Dordas and Sioulas, 2008).

Given that nitrate reduction is the rate limiting step for $\mathrm{N}$ assimilation, nitrate reductase (NR) is considered a key enzyme in $\mathrm{N}$ acquisition. NR reduces nitrate to nitrite, but is also able to generate NO from nitrite (Kaiser et al., 2010). More recently, NR has been shown to play a role in NO homeostasis by supplying electrons from $\mathrm{NAD}(\mathrm{P}) \mathrm{H}$ through its diaphorase/dehydrogenase domain both to a truncated phytoglobins, which scavenges NO by its dioxygenase activity, and to the molybdoenzyme NOforming nitrite reductase that can also generate $\mathrm{NO}$ from nitrite (Chamizo-Ampudia et al., 2017). In addition, a Nitrite:NO reductase was characterized as a membrane-bound enzyme that specifically produces NO from nitrite at pH 6 (Stöhr et al., 2001). It may reduce the apoplastic nitrite produced by NR playing a role in nitrate signaling via NO formation (Stöhr et al., 2001).

$\mathrm{NO}$ is a widespread signal molecule that participates in many physiological processes in all life kingdoms. In animals, $\mathrm{NO}$ is produced by the enzyme NO synthase (NOS; EC 1.14.13.39). All NOS make use of l-arginine and molecular oxygen as substrates and require the reduced cofactors nicotinamide-adenine-dinucleotide phosphate (NADPH), flavin adenine dinucleotide (FAD), flavin mononucleotide (FMN), and (6R-)5,6,7,8-tetrahydrobiopterin $\left(\mathrm{BH}_{4}\right)$. Animal NOS is a bimodal enzyme, comprising an $\mathrm{N}$-terminal oxygenase domain (NOSoxy) that binds protoporphyrin IX (heme) and C-terminal reductase domain (NOSred) that binds NADPH and the cofactors FMN and FAD. The two domains are connected by a calmodulin binding sequence (Griffith, 1995).

Genomic and functional analyses indicate that NOS enzymes are present in many organisms ranging from bacteria to humans (Gorren and Mayer, 2007). In higher plants, there are at least two enzymatic ways leading to NO production, the reductive and oxidative pathways: (1) NR, which reduces nitrate to nitrite, and then nitrite to NO (Yamasaki et al., 1999) and (2) a NOSlike enzymatic activity (Corpas et al., 2006). Nevertheless, no gene or protein with sequence similarity to animal or bacterial NOS has been yet identified in higher plants (Jeandroz et al., 2016). The first NOS of the plant kingdom was described in the photosynthetic unicellular Chlorophyte Ostreococcus tauri (Foresi et al., 2010; Weisslocker-Schaetzel et al., 2017). O. tauri is a single-celled green alga who shares a common ancestor with higher plants and is considered part of an early diverging class within the green plant lineage. Thus, it is an appropriate model system to study gene evolution and cellular processes in photosynthetic eukaryotes (Derelle et al., 2006).

In a previous work, we expressed OtNOS in Arabidopsis under the regulation of a stress-inducible promoter and showed that the transgenic lines displayed improved tolerance against salt and drought stresses (Foresi et al., 2015). The relevance of NO as a mediator of physiological and stress-related processes in plants has recently been reviewed (Begara-Morales et al., 2018). Meanwhile, other reports demonstrated that NO can regulate the first steps of $\mathrm{N}$ assimilation (Sanz-Luque et al., 2013) and also, through overexpression of phytoglobins, that NO can be oxidized to $\mathrm{NO}_{3}{ }^{-}$and enter into $\mathrm{N}$ assimilation pathways in dicots and monocots (Kuruthukulangarakoola et al., 2017; Zhang et al., 2019). In this work, based on the molecular characterization of OtNOS activity and NO involvement in $\mathrm{N}$ metabolism, we generated transgenic tobacco plants expressing OtNOS under the control of the Cauliflower Mosaic Virus (CaMV) 35S promoter, and analyzed their growth and response to sufficient and deficient $\mathrm{N}$ conditions. Transgenic tobacco lines expressing OtNOS exhibited higher growth rates than plants transformed with the empty vector (EV) in nitrate sufficient condition. These findings extend our knowledge about the physiology of NO and nitrate in plants.

\section{MATERIALS AND METHODS}

\section{Preparation and Characterization of Nicotiana tabacum Lines Expressing OtNOS}

The $O$. tauri DNA sequence encoding NOS was synthesized, sequenced and cloned into the Bam HI and $\mathrm{XbaI}$ sites of pCHF3 (Jarvis et al., 1998) to give pCHF3:OtNOS. Plasmid pCHF3 is a binary vector carrying the CaMV 35 S promoter and a pea (Pisum sativum L.) rubisco small subunit terminator. Nicotiana tabacum plants were transformed as described by Gallois and Marinho (1995) using Agrobacterium tumefaciens containing the plasmid of interest. Seedlings of N. tabacum cultivar Petit Havana (PH) from 6 to 8 weeks grown in Magenta boxes with MS-0, $0.8 \%$ (w/v) agar were used. After incubation and transformation process, stems of 1 to $2 \mathrm{~cm}$ long were transferred to Magenta boxes with MS-0, $0.8 \%(\mathrm{w} / \mathrm{v})$ agar containing $0.1 \mathrm{mg} \mathrm{m}^{-1}$ Kanamycin (Kan). Stems that formed roots were subsequently transferred to pots with soil.

Transgenic 35S:OtNOS lines were selected on the basis of Kan resistance, and confirmed by genomic PCR and RT-PCR analyses. Primers used for RT-PCR are specified in Supplementary Table S1 and amplified a 632-bp fragment. The primers for elongation factor $1 \alpha(e f-1 \alpha)$, a housekeeping gene used as a reference for mRNA levels between samples, are also indicated in Supplementary Table S1. For quantitative RT-PCR, total RNA was extracted using Trizol (Invitrogen). One $\mu \mathrm{g}$ of total RNA was used for first-strand CDNA synthesis with a M-MLV reverse transcriptase (Promega). PCR reactions were performed using Taq DNA polymerase (Invitrogen) with annealing temperature of $54^{\circ} \mathrm{C}$ and 35 cycles.

\section{Plant Growth}

Tobacco plants were grown in a culture chamber with long day photoperiod (16:8, light:darkness), temperature of $27^{\circ} \mathrm{C} / 23^{\circ} \mathrm{C}$ (light/dark cycle), $150 \mu \mathrm{E} \mathrm{m}^{-2} \mathrm{~s}^{-1}$ of light intensity and $60 \%$ humidity. For all experiments, transgenic plants of T1 generation were selected by sowing and growing for 9 days in plates containing $0.1 \mathrm{mg} \cdot \mathrm{ml}^{-1} \mathrm{Kan}$ and $0.8 \%(\mathrm{w} / \mathrm{v})$ agar supplemented with Hoagland solution (Hoagland and Arnon, 1938) or MS. For experiments under different $\mathrm{N}$ conditions, seedlings were transferred to plates containing a modified Hoagland/agar 
solution without ammonium and with $10 \mathrm{mM} \mathrm{NO}_{3}^{-}(5 \mathrm{mM}$ $\left.\mathrm{KNO}_{3}, 2.5 \mathrm{mM} \mathrm{Ca}\left(\mathrm{NO}_{3}\right)_{2}\right)$ or $0.5 \mathrm{mM} \mathrm{NO}_{3}{ }^{-}\left(\mathrm{KNO}_{3}\right)$. Growth analysis was performed using different $\mathrm{N}$ concentrations (10, 3, 1.5 , and $0.5 \mathrm{mM}$ ) in which $0.5 \mathrm{mM}$ was established as a deficient $\mathrm{N}$ condition for tobacco. When nitrate was $0.5 \mathrm{mM}$, Hoagland solution was supplemented with $\mathrm{KCl}$ and $\mathrm{CaSO}_{4}$ to maintain $\mathrm{Ca}^{+2}$ and $\mathrm{K}^{+}$levels. For experiments with more developed plants, seedlings were transferred to $200-\mathrm{cm}^{3}$ plastic pots with soil:perlite:vermiculite $(1: 1: 1)$ and irrigated with water or $10 \mathrm{mM}$ $\mathrm{KNO}_{3}$. For seed production, seedlings were transferred to 5lt pots containing perlite:vermiculite (1:1) and irrigated with modified Hoagland solution as previously described.

\section{Detection of NO and Determination of Nitrate and Protein}

Endogenous NO levels were estimated by using the NO-sensitive dye DAF-FM DA (Kojima et al., 1999; Moreau et al., 2008). Roots were observed by fluorescence microscopy and bright-field microscopy using an Eclipse E200 microscopy (Nikon). After Kan selection, seedlings were transferred for 7 days to plates containing agar and modified Hoagland solution as described above. For nitrate and protein determination, $100 \mathrm{mg}$ of seedlings were ground in liquid $\mathrm{N}_{2}$ and resuspended in $100 \mathrm{mM}$ sodium phosphate $\mathrm{pH}$ 7.4. After centrifugation at $10,000 \mathrm{~g}$ for $15 \mathrm{~min}$ at $4^{\circ} \mathrm{C}$, supernatants were used for nitrate determination as described by Cataldo et al. (1975). Samples were incubated with Salicylic acid $(50 \mathrm{mg} / \mathrm{ml})$ and reaction was stopped with $2 \mathrm{~N}$ $\mathrm{NaOH}$. Absorbance was measured at $410 \mathrm{~nm}$. Protein content were determined by the Bradford method (Bradford, 1976).

\section{Determination of Leaf Area and Root Length}

For all treatments, seedlings were photographed, total whole leaf area per plant and total length of all roots per plant were measured using Image $\mathrm{J}$ software (Image $\mathrm{J}^{1}$ ).

\section{Seed Germination}

Transgenic tobacco seeds were sown and germinated in plates containing $0.8 \%(\mathrm{w} / \mathrm{v})$ agar with Hoagland $\left(10 \mathrm{mM} \mathrm{NO}_{3}{ }^{-}\right)$, prior stratification. Germination was measured after 8 days, when the radicle pierced the seed coat (Bethke et al., 2006).

\section{Oxygen Consumption}

Oxygen consumption of adult leaves was analyzed using a Clarktype oxygen electrode. Detached leaves from tobacco $E V$ and transgenic plants from two growth conditions (water and $\mathrm{NO}_{3}{ }^{-}$), were pre-incubated for $2 \mathrm{~h}$ in the dark in the reaction buffer (10 $\mathrm{mM} \mathrm{K}_{2} \mathrm{HPO}_{4}, \mathrm{pH} 7.2,10 \mathrm{mM} \mathrm{KCl}, 5 \mathrm{mM} \mathrm{MgCl}_{2}, 0.3$ $\mathrm{M}$ mannitol) before measurements. Then, entire leaves were introduced into the chamber and oxygen consumption was measured for $10 \mathrm{~min}$. Values were normalized to fresh weight and expressed relative to the plants with maximal $\mathrm{O}_{2}$ consumption.

\footnotetext{
${ }^{1}$ http://imagej.nih.gov/ij/
}

\section{Quantitative PCR Analysis}

For quantitative RT-PCR, reactions were performed on a Step-one Real-time PCR machine from Applied Biosystems (California, United States) with Fast Universal SYBR Green Master Rox (Roche) to monitor the synthesis of double-stranded DNA. Software LinReg (Ruijter et al., 2009) was used to analyze data and relative transcript levels for each sample. Data were normalized against the levels of ef-1 $\alpha$ cDNA. The primer sequences used are listed in Supplementary Table S1. Primers for NR were designed to amplify transcript of both NR isoforms (NIA1, NIA2).

\section{Segregation Analysis}

For estimation of loci number in which T-DNA was integrated, seeds of T1 were sown in Petri plates with MS-0 and Kan and were incubated in a phytotron for 10 days. After that, sensible and resistant plants were counted. It was assumed that if the segregation proportion of Kan resistant: sensible is 3:1, the T-DNA has been inserted in a unique site of the genome (Murgia et al., 2004).

\section{Statistical Analysis}

Results are expressed as means \pm standard error. Data were analyzed using the Student's $t$-test for pairwise comparisons or ANOVA with post hoc Dunnett's method comparisons. We have developed a linear mixed-effects model, using the lme function from the nlme library in $\mathrm{R}$ software (version 3.1; R Foundation for Statistical Computing). Fixed effect was OtNOS expression, experiments and plates were treated as a random effect.

\section{RESULTS}

\section{Expression of OtNOS Gene in Tobacco}

We have previously showed that the expression of OtNOS with the control of a stress-inducible short promoter fragment (SPF) of the sunflower (Helianthus annuus) Hahb-4 gene improved stress tolerance of Arabidopsis (Foresi et al., 2015). Based on those results, we tested whether a constitutive expression of OtNOS in tobacco plant could also generate a benefit on growth parameters. Thus, OtNOS under the control of the constitutive CaMV $35 \mathrm{~S}$ promoter was cloned into the vector $\mathrm{pCHF} 3$ and used to transform tobacco (Nicotiana tabacum cv. Petit Havana) (Supplementary Figure S1A). Transgenic tobacco $\mathrm{T}_{0}$ lines expressing OtNOS were screened by genomic PCR (Supplementary Figure S1B) and RT-PCR (Supplementary Figure S1C).

NOS-expressing lines were characterized by measuring fresh weight (FW) of tobacco plants growing in pots containing perlite:vermiculite:soil (1:1:1) irrigated with water or $10 \mathrm{mM}$ nitrate. Transgenic OtNOS lines showed higher FW than EV plants after 26 and 60 days of irrigating with nitrate, while no significant differences were detected between lines when irrigated with water (Figures 1A,B).

Mitochondria are not only the cell organelles whose tasks are respiration and energy generation, but also they are sites 
of NO production and target in the electron transport chain (ETC), altering the organelle physiology. The NO production and action on various mitochondrial complexes of the ETC play a major role in NO signaling, energy metabolism and growth of plants (Gupta et al., 2018). To assess whether OtNOS expression affects mitochondrial respiration rate, $\mathrm{O}_{2}$ consumption was
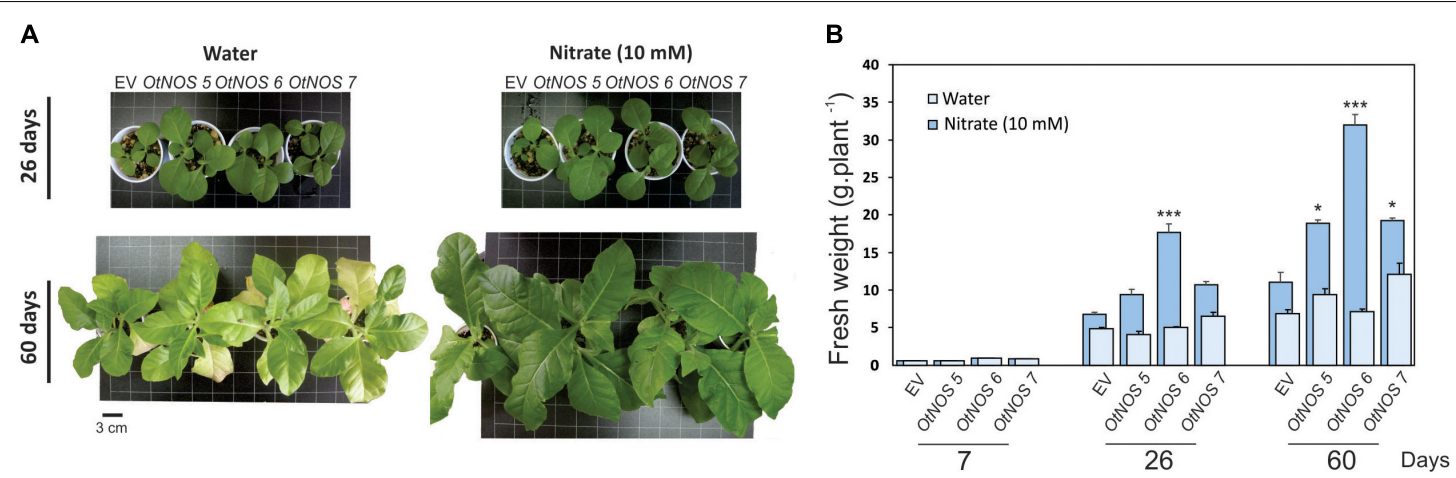

FIGURE 1 | Expression of OtNOS promotes growth of transgenic tobacco plants. (A) Representative photographs showing phenotypes of tobacco lines transformed with empty vector (EV) or OtNOS grown in plates containing MS with kanamycin (9 days) and then transferred to pots with substrate (perlite:vermiculite:soil, 1:1:1) and irrigated with water or $10 \mathrm{mM}$ nitrate for the indicated times. (B) Fresh weight of $E V$ and OtNOS transgenic lines was quantified as a measure of vegetative growth in these conditions. Values are means ( $\pm \mathrm{SE}$ ) of four to five biological replicates. Asterisks indicate statistically significant differences between OtNOS lines and EV (ANOVA post hoc Dunnett's method was used, ${ }^{*} p<0.1$ and ${ }^{* * *} p<0.001$ ).

A

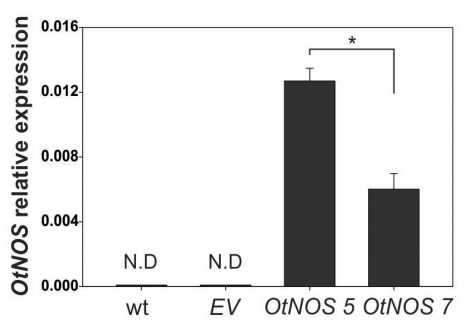

B

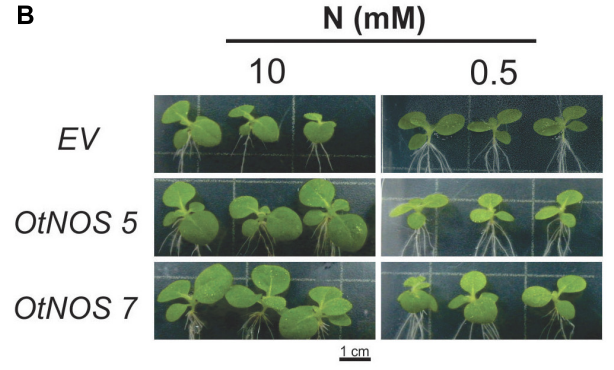

C
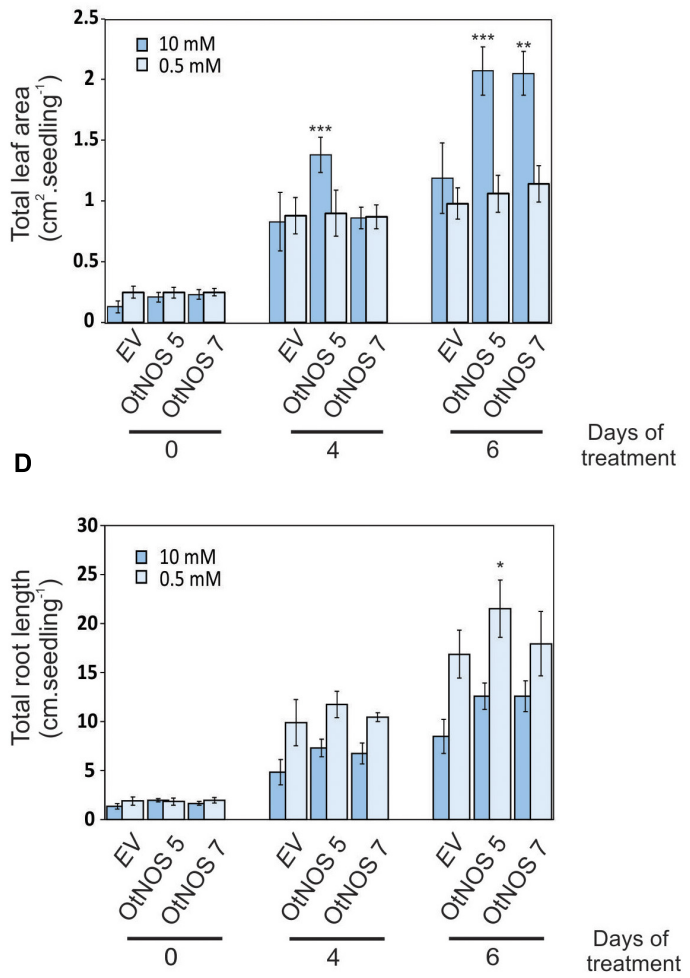

FIGURE 2 | Characterization of transgenic tobacco seedlings expressing OtNOS and growing under different N conditions. (A) Quantitative RT-PCR analysis of OtNOS expression in leaves of transgenic seedlings growing in plates containing Hoagland/agar (10 mM N) for 6 days (previously selected with kanamycin for 9 days). Values are means ( \pm SE) of three independent experiments, each consisting of a biological replicate corresponding to a pool of two to three plants. Values were relativized using ef1- $\alpha$ as control. Asterisk indicates statistically significant differences between OtNOS lines (Student's $t$-test, ${ }^{*} p<0.05$ ). N.D, no detection. (B) Representative pictures showing the phenotypes of aerial parts of 16-days-old seedlings after 6 days of growing in Hoagland medium containing 10 or 0.5 mM $\mathrm{NO}_{3}{ }^{-}$. Leaf area (C) and total root length (D) were measured during treatment using Image $\mathrm{J}$ software. Values are means $( \pm \mathrm{SE}$ ) of three independent experiments, each consisting of at least three biological replicates. Asterisks indicate statistically significant differences between OtNOS lines and seedlings transformed with empty vector (EV) in same conditions (ANOVA post hoc Dunnett's method was used, ${ }^{*} p<0.05,{ }^{* *} p<0.01,{ }^{* * *} p<0.001$ ). 
analyzed. We found that leaves of water-watered EV plants had $\sim 40 \%$ more respiration than OtNOS lines in the same condition (Supplementary Figure S2). This difference of respiration rates between OtNOS and $E V$ plants was independent of the age of the plant $(40,60$, and 70 -day old). In contrast, when plants were supplied with nitrate, less $\mathrm{O}_{2}$ consumption was observed with the increasing of the plant age and irrespective of the genotype analyzed (Supplementary Figure S2).

\section{OtNOS Expression Increases Tobacco Seedling Biomass Under N Sufficiency}

Before continuing analyzing transgenic lines, we tested the number of T-DNA insertion (Supplementary Table S2). Since more than one insertion could generate confusing results, the following experiments were done only with the OtNOS 5 and OtNOS 7 tobacco transgenic lines. First, we checked OtNOS expression by quantitative RT-PCR (qPCR) and observed that OtNOS 5 levels were two-fold higher than those of OtNOS 7 (Figure 2A). Since we observed a different growth rate between transgenic and EV plants and considering that OtNOS may affect $\mathrm{N}$ metabolism since it uses arginine as substrate, we designed an experiment using Petri dishes containing different $\mathrm{N}$ concentrations (10 and $0.5 \mathrm{mM} \mathrm{NO}_{3}{ }^{-}$). Tobacco seedlings were grown in a modified Hoagland/agar medium in which $\mathrm{NO}_{3}{ }^{-}$was used as the sole $\mathrm{N}$ source. When seedlings were grown on sufficient $\mathrm{NO}_{3}{ }^{-}$concentration $(10 \mathrm{mM})$, the OtNOS expressing seedlings exhibited growth advantages compared with EV (Figure 2B). However, when $\mathrm{N}$ was restricted $(0.5 \mathrm{mM})$ this difference was abolished (Figure 2B). Statistical analyses indicate that OtNOS expression increased leaf biomass under nitrate-rich conditions and not under $\mathrm{N}$-deficient conditions (Figures 2B,C). To get insights on root growth under contrasting nitrate availability, we measured development of the root system. Results shown in Figure 2D indicate that root length was, as expected, 50\% larger under low $\mathrm{N}$ since seedling root system tries to explore the growing media seeking for more N (Guan et al., 2014). There are no significant differences in root system growth between EV and OtNOS plants except for the transgenic line OtNOS 5 that displayed a slight increase of root growth under $\mathrm{N}$ deficiency at $0.5 \mathrm{mM}$ nitrate (Figure 2D). It has been proved that treatments with exogenous NO donors or complete blockage of endogenous NO modifies root phenotype (Correa-Aragunde et al., 2004). Since no strong effects on root architecture was observed in the transgenic tobacco lines, it is considered that levels of $\mathrm{NO}$ present in roots of tobacco OtNOS lines are below concentrations required to induce a phenotype.

\section{Levels of NO in OtNOS Transgenic Tobacco Roots}

We analyzed if the expression of OtNOS leads to an increase in NO production in tobacco plants by using the DAF-FM DA, a cell-permeable fluorescent probe that reacts with the most oxidized forms of $\mathrm{NO}\left(\mathrm{NO}^{+}\right.$and $\left.\mathrm{N}_{2} \mathrm{O}_{3}\right)$. It is the most used probe for NO detection in plants (Guo et al., 2003; Planchet and Kaiser, 2006; Zottini et al., 2007; Lozano-Juste and León, 2010;
Foresi et al., 2015). Roots of transgenic tobacco plants incubated with DAF-FM DA were analyzed by epi-florescence microscopy. After treatment, higher green fluorescence was observed in roots of both OtNOS transgenic tobacco lines compared to $E V$ (Figures 3A,B). Nicotiana tabacum corresponds to pattern type 3 of radical hairs distribution, with rows of trichoblasts and trichomes alternating with rows of atrichoblasts (Kim et al., 2006). An increase in the intensity could be seen in the zone of differentiation corresponding to the root zone where trichoblasts and trichomes are generated. However, still the apex zone is observed with fluorescence, which is especially relevant, since NO is required for primary root elongation (Sanz et al., 2014).

Results indicate that constitutive expression of OtNOS effectively increased between 25 and 30\% the NO production in tobacco root cells. To complement this observation and to get insights of the $\mathrm{NO}$ level in transgenic tobacco leaves, qPCR analysis of phytoglobin transcript level was performed considering that its transcript is induced by the increase of NO (Ohwaki et al., 2005; Shimoda et al., 2005; Kuruthukulangarakoola et al., 2017). Phytoglobins are

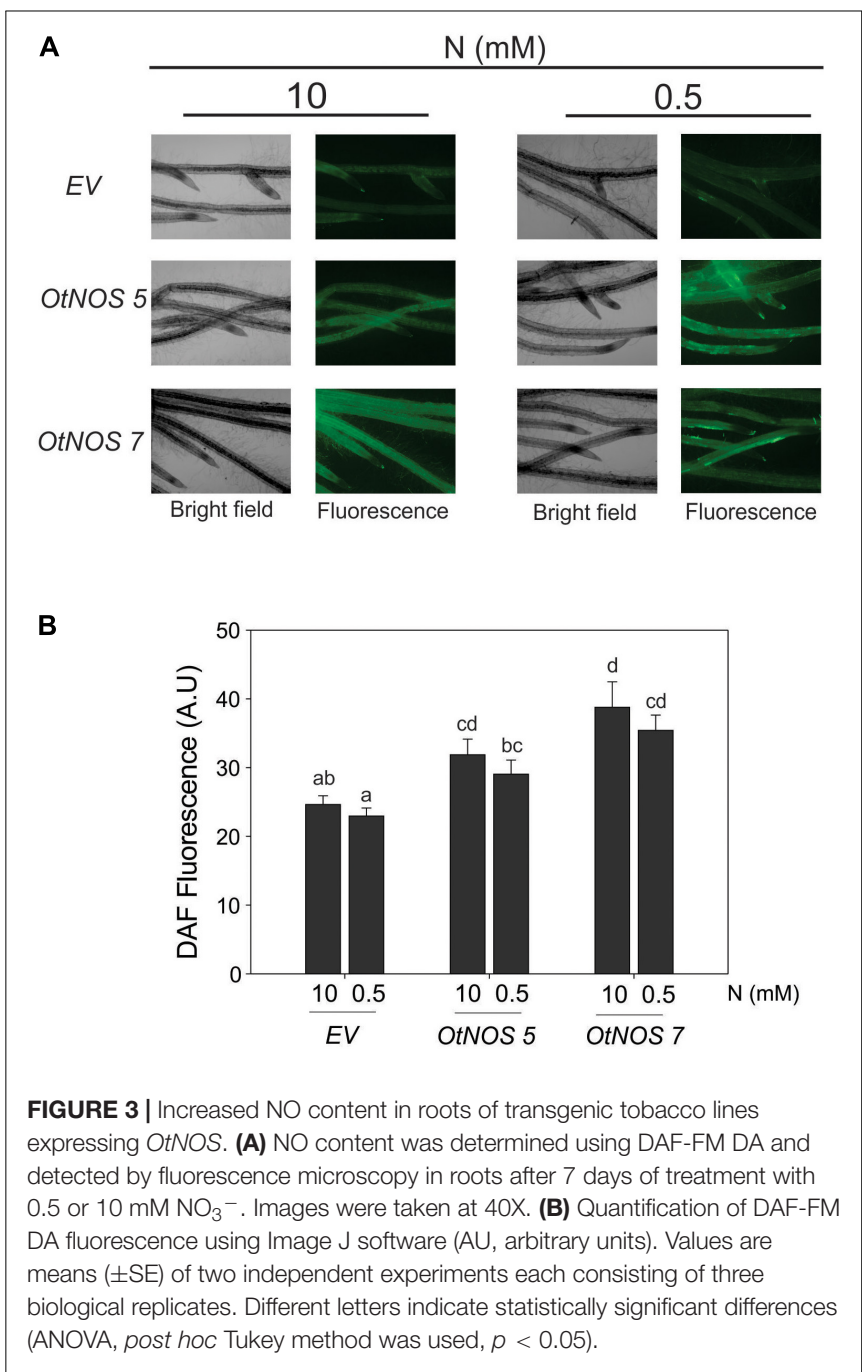



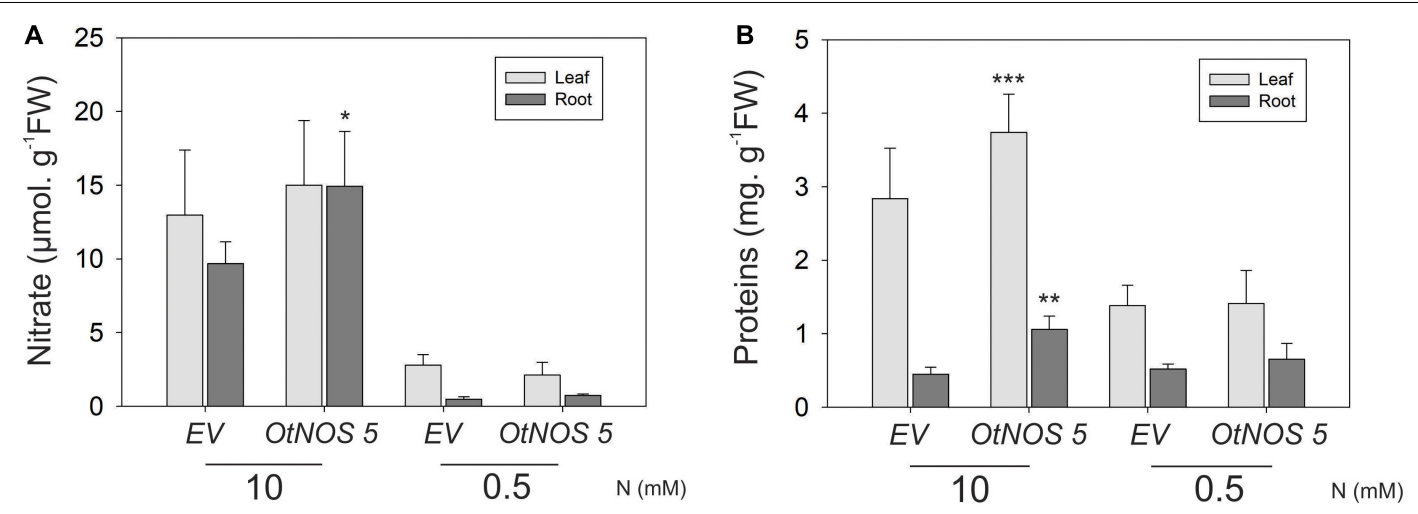

FIGURE 4 | Nitrate and protein content in transgenic tobacco line expressing OtNOS. Seedlings were grown in plates with Hoagland/agar medium containing $10 \mathrm{mM} \mathrm{NO}_{3}{ }^{-}$and kanamycin for 9 days. After selection, seedlings were transferred to plates with indicated $\mathrm{N}$ levels for 7 days for nitrate and protein determinations. Measurements were done using all leaves and root independently. (A) Nitrate was measured according to Cataldo et al. (1975). (B) Protein content was measured using Bradford's method (1976). Fresh weight (FW). Values are means ( \pm SE) of at least three independent experiments each consisting of three to four biological replicates corresponding to a pool of two to three plants. Asterisks indicate statistically significant differences between OtNOS 5 and EV (ANOVA, post hoc Dunnett's method was used, $\left.{ }^{*} p<0.05,{ }^{* *} p<0.01,{ }^{* \star *} p<0.001\right)$.

responsible of contributing to the control of high levels of NO in plants (Hebelstrup et al., 2013). In agreement with results obtained using the NO probe in roots, transcript levels of tobacco phytoglobin showed a slight increase in leaves of both transgenic tobacco lines OtNOS 5 and OtNOS 7 (Supplementary Figure S3).

\section{Higher Expression of Nitrate Reductase (NR) in Tobacco OtNOS Transgenic Line}

The growth phenotype of OtNOS transgenic tobacco plants prompted us to further explore $\mathrm{N}$ metabolite assimilation in those lines. Nitrate and protein content were measured from leaves and roots of OtNOS 5 and $E V$ tobacco lines. While no differences were found under low $\mathrm{N}$ condition, OtNOS 5 line increases the levels of nitrate and protein compared to $E V$ when grown in $\mathrm{N}$ sufficient condition (Figure 4). Accordingly, OtNOS 7 line also increased protein content in leaves and roots during $\mathrm{N}$ sufficiency (Supplementary Figure S4).

To further investigate nitrate assimilation pathway, levels of NR transcript were studied in a short time of treatment since it is known that genes responding to nitrogen are highly dependent on $\mathrm{N}$ availability (Liu et al., 2017). Given that transgenic tobacco lines displayed similar growth phenotype compared to $E V$ under low $\mathrm{N}$ conditions, we only analyzed $\mathrm{NR}$ expression under complete N supply. Results show that NR expression is higher in OtNOS 5 and 7 compared to EV (Figure 5).

\section{OtNOS Expression Might Promote Tobacco Seed Yield When N Is Sufficient}

It is well known that seed production is highly dependent on the plant N status (Sinclair and Rufty, 2012). To analyze whether $\mathrm{N}$ availability modifies differentially the number of flowers and seed production in transgenic OtNOS plants, transgenic and $E V$ plants were grown until the end of life cycle. A positive effect of OtNOS expression could be observed in the number of flowers and seed yield under complete $\mathrm{N}$ condition in the

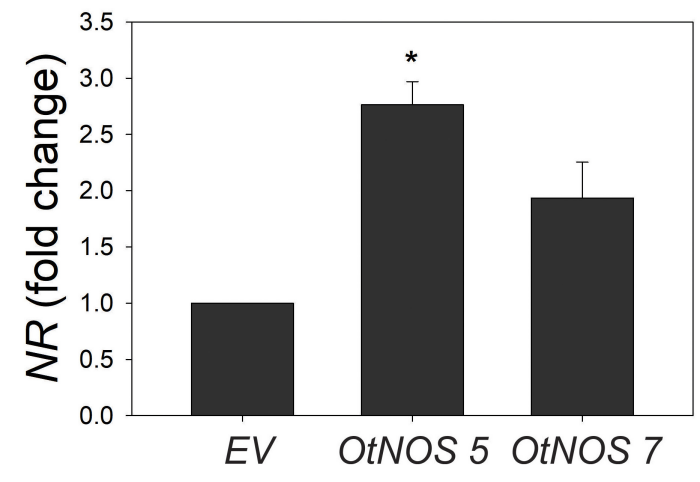

FIGURE 5 | Transcript levels of nitrate reductase (NR). Seedlings were grown in plates with Hoagland medium containing $10 \mathrm{mM} \mathrm{NO}_{3}{ }^{-}$and kanamycin for 9 days. After selection, seedlings were transferred to plates with the same $\mathrm{N}$ condition for 3 days and quantitative RT-PCR analysis of $N R$ transcript level was perfomed. NR expression was relativized to EV plants. Values are means $( \pm \mathrm{SE}$ ) of three independent experiments, each consisting of three biological replicates corresponding to a pool of two to three plants. Asterisk indicates statically significant differences compare to EV (Student's $t$-test, ${ }^{\star} p<0.05$ ).

line OtNOS 5 (Table 1), that correlates with a high level of OtNOS expression. This effect was prevented when $\mathrm{N}$ supply was deficient (Table 1). Results indicate that $\mathrm{N}$ restriction resulted in a severe attenuation of the OtNOS-promoted stimulation of growth and production in tobacco.

\section{DISCUSSION}

Optimal plant growth fully relies on the availability of soil nutrients. Nitrogen $(\mathrm{N})$ is a central resource required in large amounts to sustain the synthesis of organic molecules that constitute the plant. Arginine is not only a building block for protein biosynthesis, but also contains the highest nitrogen to carbon $(\mathrm{N} / \mathrm{C})$ ratio among amino acids, being a storage molecule 
TABLE 1 | Transgenic tobacco line OtNOS5 showed increased seed yield under complete nitrogen supply.

\begin{tabular}{|c|c|c|c|c|c|c|}
\hline & $\begin{array}{c}\text { Nitrogen } \\
(\mathrm{mM})\end{array}$ & $E V^{a}$ & OtNOS $5^{b}$ & Ratio b/a & OtNOS $7^{c}$ & Ratio $\mathrm{c/a}$ \\
\hline \multirow[t]{2}{*}{ Flowers. plant ${ }^{-1}$} & 10 & $30 \pm 2$ & $47 \pm 4$ & $1.5^{\star \star}$ & 33 & 1.1 \\
\hline & 0.5 & $16 \pm 1$ & $18 \pm 2$ & 1.1 & $15 \pm 1$ & 0.9 \\
\hline \multirow[t]{2}{*}{ Total seed weight. plant ${ }^{-1}(\mathrm{~g})$} & 10 & $1.32 \pm 0.2$ & $2.38 \pm 0.5$ & $1.8^{*}$ & 1.0 & 0.8 \\
\hline & 0.5 & $0.64 \pm 0.02$ & $0.71 \pm 0.12$ & 1.1 & $0.63 \pm 0.01$ & 0.9 \\
\hline \multirow[t]{2}{*}{ Individual seed weight (mg) } & 10 & 0.067 & 0.079 & 1.2 & 0.094 & 1.4 \\
\hline & 0.5 & 0.058 & 0.069 & 1.2 & 0.068 & 1.2 \\
\hline \multirow[t]{2}{*}{ Germination (\%) } & 10 & 96 & 98 & 1.02 & 78 & 0.81 \\
\hline & 0.5 & 67 & 68 & 1.02 & 33 & 0.50 \\
\hline
\end{tabular}

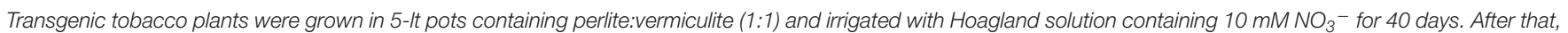

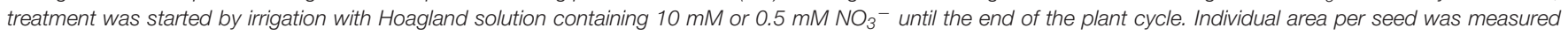

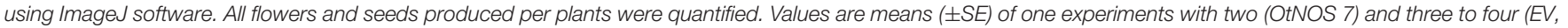

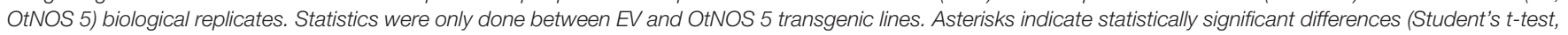
$\left.{ }^{*} p<0.1,{ }^{* *} p<0.05\right)$.

of organic $\mathrm{N}$ in plant cells. Thus, arginine metabolism possesses many physiological implications in higher plants (Winter et al., 2015). Plant arginases degrade arginine to produce ornithine and urea (Meng et al., 2015). Arginine is also a substrate for NO synthesis in plants through an activity of a yet undescribed protein named NO synthase-like (NOS-like) (Gas et al., 2009). We have previously shown that transgenic expression of OtNOS in Arabidopsis resulted in positive effects on germination, aerial growth and responses to water deficit mediated by changes in stomatal index and pore aperture (Foresi et al., 2015).

In this work, we show that transgenic tobacco plants expressing OtNOS are able to grow faster than siblings transformed with the $E V$. In addition, the transgenic line OtNOS 5 , expressing higher level of OtNOS than OtNOS 7 line is able to generate up to $80 \%$ more seeds than OtNOS 7 and $E V$ tobacco plants. In another work, transgenic tobacco plants that over expressed a mammalian NOS were generated (Chun et al., 2012). These transgenic plants are smaller compared to the wild type, exhibited enhanced resistance to biotic stress and contained high level of salicylic acid. Mammalian NOS uses the cofactor $\mathrm{BH}_{4}$, but there are no biosynthetic pathways described for this cofactor in plants. Unlike OtNOS, that belongs to the plant kingdom and is capable of using tetrahydrofolate (THF) as cofactor, mammalian NOS activity was not detected with THF (Adak et al., 2002). Thereby, more work is necessary to understand how mammalian NOS activity is generating NO in the transformed tobacco plants. Here, it was demonstrated that OtNOS expression could bring potential benefits improving $\mathrm{N}$ metabolism in tobacco, a plant strongly dependent on $\mathrm{N}$ supply (Ruiz et al., 2006). Three experimental conditions were assayed to analyze the effects of OtNOS expression on tobacco growing under sufficient and deficient $\mathrm{N}$ supply: (i) pots with limited soil content supplemented or not with $\mathrm{NO}_{3}{ }^{-}$, (ii) plates containing 0.5 or $10 \mathrm{mM} \mathrm{NO}_{3}{ }^{-}$and (iii) pots containing perlite:vermiculite (1:1) and irrigated with Hoagland containing 0.5 or $10 \mathrm{mM}$ $\mathrm{NO}_{3}{ }^{-}$. In all experimental models, OtNOS expression conferred tobacco an enhanced growth under sufficient $\mathrm{N}$ availability.

Mitochondria are tightly linked to $\mathrm{N}$ metabolism and assimilation in plants (Szal and Podgórska, 2012) and is also a source of NO generated from nitrite and cytochrome $\mathrm{c}$ oxidoreductase activity in complex III (Alber et al., 2017). Additionally, NO was shown to be able to partially inhibit mitochondrial respiration (Gupta et al., 2018). According to this, under low $\mathrm{N}$ condition, OtNOS transgenic plants display $\sim 40 \%$ inhibition of mitochondrial respiration respect to $E V$ plants. In other reports, it has been shown that NO could inhibit aconitase, induce alternative oxidase (Kumari et al., 2019) and shift the metabolism toward amino acid and protein synthesis (Cvetkovska and Vanlerberghe, 2012; Gupta et al., 2012). We showed that transgenic tobacco lines have no different respiration rates under sufficient $\mathrm{N}$ availability. In this sense, it cannot be ascribed OtNOS phenotype to different respiration rates since the increase growth, nitrate and protein content in the OtNOS transgenic lines was observed only when plants where supplemented with nitrate.

NR expression was higher in the transgenic line OtNOS 5 than in OtNOS 7, and it correlates with a higher OtNOS expression in OtNOS 5. NR expression and activity are regulated by many factors, nitrate, light, phytohormones, low temperature, drought, among others (Lillo et al., 2003; Lea et al., 2004; Park et al., 2011). Furthermore, NR is not only a key enzyme for $\mathrm{N}$ acquisition and assimilation in plants (Campbell, 2001), but also a key enzyme to modulate plant NO homeostasis (Tejada-Jimenez et al., 2019). The double mutant NR (nia1 nia2) has decrease nitrite levels and impaired NO synthesis (Modolo et al., 2006). These evidences indicate that there is a mutual regulation between NR activity and $\mathrm{NO}$, hindering the interpretation of the results.

The NR substrate $\mathrm{NO}_{3}{ }^{-}$has also been shown to regulate flowering induction (Marín et al., 2011), crop growth and yield increments (Lawlor, 2002; Krapp et al., 2014). Moreover, the over-expression of tobacco $N R$ increases seed protein content and weight in wheat without increasing the $\mathrm{N}$ supply (Zhao et al., 2013). We report here that the transgenic tobacco line that express more OtNOS transcript levels presented a positive correlation with protein content, NR expression and seed production. However, when $\mathrm{N}$ has been restricted, the promoter effect of OtNOS was blocked. Under $\mathrm{N}$ deficient conditions, it can be detected similar levels of OtNOS expression as in N sufficiency (Supplementary Figure S5) but only a slight decrease in NO content. The lack of correlation between lines for OtNOS 
transcript levels, NO production and the growth phenotype may suggest that multiple factors are involved. Indeed, the effects observed in OtNOS lines may be not only due to the increment of $\mathrm{NO}$, but also a result of NOS activity such as arginine depletion and/or increase of citrulline levels. Furthermore, NO homeostasis in plants is regulated through many oxidation processes and enzymatic activities (e.g. phytoglobin activity, chemical reaction with proteins and redox state of the cell).

As stated, NUE is an attractive target to modulate and improve growth and yield in crop plants. Recent findings in cyanobacteria highlight the importance of how $\mathrm{N}$ metabolism can be enriched through the activity of new and unexpected enzymes such as a singular NOS with a globin domain found in Synechoccocus PCC 7335 (SyNOS) (Correa-Aragunde et al., 2018) and arginine dihydrolase found in Synechocystis sp. PCC 6803 (Zhang et al., 2018). SyNOS and arginine dihydrolase metabolize arginine to generate mainly nitrate and ammonia, respectively, which are able to re-enter in the first steps of $\mathrm{N}$ assimilation and supply the elementary blocks required for the synthesis of macromolecules. Increasing the ability of plant cells for recycling macronutrients from storage molecules seems to be a promissory novel strategy to attain the goal of diminishing fertilization practices in crop fields without affecting yield.

\section{DATA AVAILABILITY STATEMENT}

The datasets generated for this study are available on request to the corresponding author.

\section{REFERENCES}

Adak, S., Aulak, K. S., and Stuehr, D. J. (2002). Direct evidence for nitric oxide production by a nitric-oxide synthase-like protein from Bacillus subtilis. J. Biol. Chem. 277, 16167-16171. doi: 10.1074/jbc.M201136200

Alber, N. A., Sivanesan, H., and Vanlerberghe, G. C. (2017). The occurrence and control of nitric oxide generation by the plant mitochondrial electron transport chain. Plant Cell Environ. 40, 1074-1085. doi: 10.1111/pce.12884

Begara-Morales, J. C., Chaki, M., Valderrama, R., Sánchez-Calvo, B., Mata-Pérez, C., Padilla, M. N., et al. (2018). Nitric oxide buffering and conditional nitric oxide release in stress response. J. Exp. Bot. 69, 3425-3438. doi: 10.1093/jxb/ ery072

Bethke, P. C., Libourel, I. G. L., and Jones, R. L. (2006). Nitric oxide reduces seed dormancy in Arabidopsis. J. Exp. Bot. 57, 517-526. doi: 10.1093/jxb/erj060

Bradford, M. M. (1976). A rapid and sensitive method for the quantitation of protein utilizing the principle of protein-dye binding. Anal. Biochem. 72, 248-254. doi: 10.1016/0003-2697(76)90527-3

Campbell, W. H. (2001). Structure and function of eukaryotic NAD(P)H:Nitrate reductase. Cell. Mol. Life Sci. 58, 194-204. doi: 10.1007/PL00000847

Cataldo, D. A., Maroon, M., Schrader, L. E., and Youngs, V. L. (1975). Rapid colorimetric determination of nitrate in plant tissue by nitration of salicylic acid. Commun. Soil Sci. Plant Anal. 6, 71-80. doi: 10.1080/00103627509366547

Chamizo-Ampudia, A., Sanz-Luque, E., Llamas, A., Galvan, A., and Fernandez, E. (2017). Nitrate reductase regulates plant nitric oxide homeostasis. Trends Plant Sci. 22, 163-174. doi: 10.1016/j.tplants.2016.12.001

Chun, H. J., Park, H. C., Koo, S. C., Lee, J. H., Park, C. Y., Choi, M. S., et al. (2012). Constitutive expression of mammalian nitric oxide synthase in tobacco plants triggers disease resistance to pathogens. Mol. Cells 34, 463-471. doi: 10.1007/s10059-012-0213-0

\section{AUTHOR CONTRIBUTIONS}

NF, AL, NC-A, NC, and LL are members of the research staff. AN and FD are graduates fellows and MM is a postdoctoral fellow from CONICET, Argentina.

\section{FUNDING}

This research was supported by the Agencia Nacional de Promoción Científica y Tecnológica (ANPCyT, PICTs 2927/2015, PICT-2015-3828, and PICT-2014-2496 to LL, NC-A, and $\mathrm{AL}$, respectively), Consejo Nacional de Investigaciones Científicas y Técnicas (CONICET; PIP 0903/2011 to LL), and institutional grants from the Universidad Nacional de Mar del Plata, Argentina.

\section{ACKNOWLEDGMENTS}

We thank Dr. Cristina Lombardo (National University of Mar del Plata-CONICET), for the critical analysis of the tobacco root fluorescence images.

\section{SUPPLEMENTARY MATERIAL}

The Supplementary Material for this article can be found online at: https://www.frontiersin.org/articles/10.3389/fpls.2020.00312/ full\#supplementary-material

Corpas, F. J., Barroso, J. B., Carreras, A., Valderrama, R., Palma, J. M., León, A. M., et al. (2006). Constitutive arginine-dependent nitric oxide synthase activity in different organs of pea seedlings during plant development. Planta 224, 246-254. doi: 10.1007/s00425-005-0205-9

Correa-Aragunde, N., Foresi, N., Del Castello, F., and Lamattina, L. (2018). A singular nitric oxide synthase with a globin domain found in Synechococcus PCC 7335 mobilizes N from arginine to nitrate. Sci. Rep. 8:12505. doi: 10.1038/ s41598-018-30889-6

Correa-Aragunde, N., Graziano, M., and Lamattina, L. (2004). Nitric oxide plays a central role in determining lateral root development in tomato. Planta 218, 900-905. doi: 10.1007/s00425-003-1172-7

Cvetkovska, M., and Vanlerberghe, G. C. (2012). Alternative oxidase modulates leaf mitochondrial concentrations of superoxide and nitric oxide. New Phytol. 195, 32-39. doi: 10.1111/j.1469-8137.2012.04166.x

Derelle, E., Ferraz, C., Rombauts, S., Rouzé, P., Worden, A. Z., Robbens, S., et al. (2006). Genome analysis of the smallest free-living eukaryote Ostreococcus tauri unveils many unique features. Proc. Natl. Acad. Sci. U.S.A. 103, 11647-11652. doi: 10.1073/pnas.0604795103

Dordas, C. A., and Sioulas, C. (2008). Safflower yield, chlorophyll content, photosynthesis, and water use efficiency response to nitrogen fertilization under rainfed conditions. Ind. Crops Prod. 27, 75-85. doi: 10.1016/j.indcrop.2007.07. 020

Foresi, N., Correa-Aragunde, N., Parisi, G., Caló, G., Salerno, G., and Lamattina, L. (2010). Characterization of a nitric oxide synthase from the plant kingdom: no generation from the green alga Ostreococcus tauri is light irradiance and growth phase dependent. Plant Cell 22, 3816-3830. doi: 10.1105/tpc.109.073510

Foresi, N., Mayta, M. L., Lodeyro, A. F., Scuffi, D., Correa-Aragunde, N., GarcíaMata, C., et al. (2015). Expression of the tetrahydrofolate-dependent nitric oxide synthase from the green alga Ostreococcus tauri increases tolerance to 
abiotic stresses and influences stomatal development in Arabidopsis. Plant J. 82, 806-821. doi: 10.1111/tpj.12852

Gallois, P., and Marinho, P. (1995). Leaf disk transformation using Agrobacterium tumefaciens-expression of heterologous genes in tobacco. Plant Gene Transf. Expr. Protoc. 49, 39-48. doi: 10.1385/0-89603-321-X:39

Gas, E., Flores-Pérez, Ú., Sauret-Güeto, S., and Rodríguez-Concepción, M. (2009). Hunting for plant nitric oxide synthase provides new evidence of a central role for plastids in nitric oxide metabolism. Plant Cell 21, 18-23. doi: 10.1105/tpc. 108.065243

Gorren, A. C. F., and Mayer, B. (2007). Nitric-oxide synthase: a cytochrome P450 family foster child. Biochim. Biophys. Acta Gen. Subj. 1770, 432-445. doi: 10. 1016/j.bbagen.2006.08.019

Griffith, O. (1995). Nitric oxide synthases: properties and catalytic mechanism. Annu. Rev. Physiol. 57, 707-736. doi: 10.1146/annurev.physiol.57.1.707

Guan, P., Wang, R., Nacry, P., Breton, G., Kay, S. A., Pruneda-Paz, J. L., et al. (2014). Nitrate foraging by Arabidopsis roots is mediated by the transcription factor TCP20 through the systemic signaling pathway. Proc. Natl. Acad. Sci. U.S.A. 111, 15267-15272. doi: 10.1073/pnas.1411375111

Guo, F. Q., Okamoto, M., and Crawford, N. M. (2003). Identification of a plant nitric oxide synthase gene involved in hormonal signaling. Science 302, 100103. doi: $10.1126 /$ science. 1086770

Gupta, K. J., Kumari, A., Florez-Sarasa, I., Fernie, A. R., and Igamberdiev, A. U. (2018). Interaction of nitric oxide with the components of the plant mitochondrial electron transport chain. J. Exp. Bot. 69, 3413-3424. doi: 10.1093/ jxb/ery119

Gupta, K. J., Shah, J. K., Brotman, Y., Jahnke, K., Willmitzer, L., Kaiser, W. M., et al. (2012). Inhibition of aconitase by nitric oxide leads to induction of the alternative oxidase and to a shift of metabolism towards biosynthesis of amino acids. J. Exp. Bot. 63, 1773-1784. doi: 10.1093/jxb/ers053

Hebelstrup, K. H., Shah, J. K., and Igamberdiev, A. U. (2013). The role of nitric oxide and hemoglobin in plant development and morphogenesis. Physiol. Plant. 148, 457-469. doi: 10.1111/ppl.12062

Hoagland, D. R., and Arnon, D. I. (1938). The Water-Culture Method for Growing Plants without Soil. Berkeley, CA: University of California.

Huber, S. C., Sugiyama, T., and Alberte, R. S. (1989). Photosynthetic determinants of growth in maize plants: effects of nitrogen nutrition on growth, carbon fixation and photochemical features. Plant Cell Physiol. 30, 1063-1072. doi: 10.1093/oxfordjournals.pcp.a077846

Jarvis, P., Chen, L. J., Li, H. M., Peto, C. A., Fankhauser, C., and Chory, J. (1998). An Arabidopsis mutant defective in the plastid general protein import apparatus. Science 282, 100-103. doi: 10.1126/science.282.5386.100

Jeandroz, S., Wipf, D., Stuehr, D. J., Lamattina, L., Melkonian, M., Tian, Z., et al. (2016). Occurrence, structure, and evolution of nitric oxide synthase-like proteins in the plant kingdom. Sci. Signal. 9:re2. doi: 10.1126/scisignal.aad4403

Kaiser, W. M., Planchet, E., and Rümer, S. (2010). Nitrate reductase and nitric oxide. Nitrogen Metab. Plants Post Genomic Era 42, 127-145. doi: 10.1002/ 9781444328608.ch6

Kim, D. W., Lee, S. H., Choi, S. B., Won, S. K., Heo, Y. K., Cho, M., et al. (2006). Functional conservation of a root hair cell-specific cis-element in angiosperms with different root hair distribution patterns. Plant Cell 18, 2959-2970. doi: $10.1105 /$ tpc. 106.045229

Kojima, H., Urano, Y., Kikuchi, K., Higuchi, T., Hirata, Y., and Nagano, T. (1999). Fluorescent indicators for imaging nitric oxide production. Angew. Chem. Int. Ed. 38, 3209-3212. doi: 10.1002/(sici)1521-3773(19991102)38:21<3209::aidanie3209>3.0.co;2-6

Krapp, A., David, L. C., Chardin, C., Girin, T., Marmagne, A., Leprince, A. S., et al. (2014). Nitrate transport and signalling in Arabidopsis. J. Exp. Bot. 65, 789-798. doi: $10.1093 / \mathrm{jxb} / \mathrm{eru} 001$

Krstić, B., and Sarić, M. R. (1983). Efficiency of nitrogen utilization and photosynthetic rate in C3 and C4 plants. Genet. Asp. Plant Nutr. 106, 255-260. doi: 10.1007/978-94-009-6836-3_31

Kumari, A., Pathak, P. K., Bulle, M., Igamberdiev, A. U., and Gupta, K. J. (2019). Alternative oxidase is an important player in the regulation of nitric oxide levels under normoxic and hypoxic conditions in plants. J. Exp. Bot. 70, 4345-4354. doi: $10.1093 / \mathrm{jxb} / \mathrm{erz} 160$

Kuruthukulangarakoola, G. T., Zhang, J., Albert, A., Winkler, B., Lang, H., Buegger, F., et al. (2017). Nitric oxide-fixation by non-symbiotic haemoglobin proteins in
Arabidopsis thaliana under N-limited conditions. Plant Cell Environ. 40, 36-50. doi: 10.1111/pce.12773

Lawlor, D. W. (2002). Carbon and nitrogen assimilation in relation to yield: mechanisms are the key to understanding production systems. J. Exp. Bot. 53, 773-787. doi: 10.1093/jxb/53.370.773

Lea, U. S., Ten Hoopen, F., Provan, F., Kaiser, W. M., Meyer, C., and Lillo, C. (2004). Mutation of the regulatory phosphorylation site of tobacco nitrate reductase results in high nitrite excretion and NO emission from leaf and root tissue. Planta 219, 59-65. doi: 10.1007/s00425-004-1209-6

Lillo, C., Lea, U. S., Leydecker, M. T., and Meyer, C. (2003). Mutation of the regulatory phosphorylation site of tobacco nitrate reductase results in constitutive activation of the enzyme in vivo and nitrite accumulation. Plant J. 35, 566-573. doi: 10.1046/j.1365-313X.2003.01828.x

Liu, K., Niu, Y., Konishi, M., Wu, Y., Du, H., Chung, H. S., et al. (2017). Discovery of nitrate-CPK-NLP signalling in central nutrient-growth networks. Nature 545, 311-316. doi: 10.1038/nature22077

Lozano-Juste, J., and León, J. (2010). Enhanced abscisic acid-mediated responses in nialnia2noal-2 triple mutant impaired in NIA/NR- and AtNOA1-dependent nitric oxide biosynthesis in Arabidopsis. Plant Physiol. 152, 891-903. doi: 10. 1104/pp.109.148023

Marín, I. C., Loef, I., Bartetzko, L., Searle, I., Coupland, G., Stitt, M., et al. (2011). Nitrate regulates floral induction in Arabidopsis, acting independently of light, gibberellin and autonomous pathways. Planta 233, 539-552. doi: 10.1007/ s00425-010-1316-5

Masclaux-Daubresse, C., Daniel-Vedele, F., Dechorgnat, J., Chardon, F., Gaufichon, L., and Suzuki, A. (2010). Nitrogen uptake, assimilation and remobilization in plants: challenges for sustainable and productive agriculture. Ann. Bot. 105, 1141-1157. doi: 10.1093/aob/mcq028

Meng, Z., Meng, Z., Zhang, R., Liang, C., Wan, J., Wang, Y., et al. (2015). Expression of the rice arginase gene OsARG in cotton influences the morphology and nitrogen transition of seedlings. PLoS One 10:e0141530. doi: 10.1371/journal.pone.0141530

Modolo, L. V., Augusto, O., Almeida, I. M. G., Pinto-Maglio, C. A. F., Oliveira, H. C., Seligman, K., et al. (2006). Decreased arginine and nitrite levels in nitrate reductase-deficient Arabidopsis thaliana plants impair nitric oxide synthesis and the hypersensitive response to Pseudomonas syringae. Plant Sci. 171, 34-40. doi: 10.1016/j.plantsci.2006.02.010

Moreau, M., Gyu, I. L., Wang, Y., Crane, B. R., and Klessig, D. F. (2008). AtNOS/AtNOA1 is a functional Arabidopsis thaliana cGTPase and not a nitricoxide synthase. J. Biol. Chem. 283, 32957-32967. doi: 10.1074/jbc.M80483 8200

Murgia, I., Tarantino, D., Vannini, C., Bracale, M., Carravieri, S., and Soave, C. (2004). Arabidopsis thaliana plants overexpressing thylakoidal ascorbate peroxidase show increased resistance to Paraquat-induced photooxidative stress and to nitric oxide-induced cell death. Plant J. 38, 940-953. doi: 10.1111/ j.1365-313X.2004.02092.x

Ohwaki, Y., Kawagishi-Kobayashi, M., Wakasa, K., Fujihara, S., and Yoneyama, T. (2005). Induction of class-1 non-symbiotic hemoglobin genes by nitrate, nitrite and nitric oxide in cultured rice cells. Plant Cell Physiol. 46, 324-331. doi: $10.1093 / \mathrm{pcp} / \mathrm{pci} 030$

Park, B. S., Song, J. T., and Seo, H. S. (2011). Arabidopsis nitrate reductase activity is stimulated by the E3 SUMO ligase AtSIZ1. Nat. Commun. 2:400. doi: 10.1038/ ncomms 1408

Planchet, E., and Kaiser, W. M. (2006). Nitric oxide (NO) detection by DAF fluorescence and chemiluminescence: a comparison using abiotic and biotic NO sources. J. Exp. Bot. 57, 3043-3055. doi: 10.1093/jxb/erl070

Ruijter, J. M., Ramakers, C., Hoogaars, W. M. H., Karlen, Y., Bakker, O., van den Hoff, M. J. B., et al. (2009). Amplification efficiency: linking baseline and bias in the analysis of quantitative PCR data. Nucleic Acids Res. 37:e45. doi: 10.1093/nar/gkp045

Ruiz, J. M., Rivero, R. M., Cervilla, L. M., Castellano, R., and Romero, L. (2006). Grafting to improve nitrogen-use efficiency traits in tobacco plants. J. Sci. Food Agric. 86, 1014-1021. doi: 10.1002/jsfa.2450

Sanz, L., Fernández-Marcos, M., Modrego, A., Lewis, D. R., Muday, G. K., Pollmann, S., et al. (2014). Nitric oxide plays a role in stem cell niche homeostasis through its interaction with auxin. Plant Physiol. 166, 1972-1984. doi: $10.1104 /$ pp.114.247445 
Sanz-Luque, E., Ocaña-Calahorro, F., Llamas, A., Galvan, A., and Fernandez, E. (2013). Nitric oxide controls nitrate and ammonium assimilation in Chlamydomonas reinhardtii. J. Exp. Bot. 64, 3373-3383. doi: 10.1093/jxb/ert175

Shimoda, Y., Nagata, M., Suzuki, A., Abe, M., Sato, S., Kato, T., et al. (2005). Symbiotic rhizobium and nitric oxide induce gene expression of non-symbiotic hemoglobin in Lotus japonicus. Plant Cell Physiol. 46, 99-107. doi: 10.1093/pci/ pci001

Sinclair, T. R., and Rufty, T. W. (2012). Nitrogen and water resources commonly limit crop yield increases, not necessarily plant genetics. Glob. Food Sec. 1, 94-98. doi: 10.1016/j.gfs.2012.07.001

Stöhr, C., Strube, F., Marx, G., Ullrich, W. R., and Rockel, P. (2001). A plasma membrane-bound enzyme of tobacco roots catalyses the formation of nitric oxide from nitrite. Planta 212, 835-841. doi: 10.1007/s00425000 0447

Szal, B., and Podgórska, A. (2012). The role of mitochondria in leaf nitrogen metabolism. Plant Cell Environ. 35, 1756-1768. doi: 10.1111/j.1365-3040.2012. 02559.x

Tejada-Jimenez, M., Llamas, A., Galván, A., and Fernández, E. (2019). Role of nitrate reductase in NO production in photosynthetic eukaryotes. Plants 8:56. doi: 10.3390/plants8030056

Tóth, V. R., Mészáros, I., Veres, S., and Nagy, J. (2002). Effects of the available nitrogen on the photosynthetic activity and xanthophyll cycle pool of maize in field. J. Plant Physiol. 159, 627-634. doi: 10.1078/0176-1617-0640

Weisslocker-Schaetzel, M., André, F., Touazi, N., Foresi, N., Lembrouk, M., Dorlet, P., et al. (2017). The NOS-like protein from the microalgae Ostreococcus tauri is a genuine and ultrafast NO-producing enzyme. Plant Sci. 265, 100-111. doi: $10.1016 /$ j.plantsci.2017.09.019

Winter, G., Todd, C. D., Trovato, M., Forlani, G., and Funck, D. (2015). Physiological implications of arginine metabolism in plants. Front. Plant Sci. 6:534. doi: $10.3389 /$ fpls.2015.00534
Yamasaki, H., Sakihama, Y., and Takahashi, S. (1999). An alternative pathway for nitric oxide production in plants: new features of an old enzyme. Trends Plant Sci. 4, 128-129. doi: 10.1016/S1360-1385(99)01393-X

Zhang, H., Liu, Y., Nie, X., Liu, L., Hua, Q., Zhao, G. P., et al. (2018). The cyanobacterial ornithine-ammonia cycle involves an arginine dihydrolase article. Nat. Chem. Biol. 14, 575-581. doi: 10.1038/s41589-0180038-z

Zhang, J., Buegger, F., Albert, A., Ghirardo, A., Winkler, B., Schnitzler, J.-P., et al. (2019). Phytoglobin overexpression promotes barley growth in the presence of enhanced level of atmospheric nitric oxide. J. Exp. Bot. 70, 4521-4537. doi: 10.1093/jxb/erz249

Zhao, X. Q., Nie, X. L., and Xiao, X. G. (2013). Over-expression of a tobacco nitrate reductase gene in wheat (Triticum aestivum L.) increases seed protein content and weight without augmenting nitrogen supplying. PLoS One 8:e74678. doi: 10.1371/journal.pone.0074678

Zottini, M., Costa, A., De Michele, R., Ruzzene, M., Carimi, F., and Lo Schiavo, F. (2007). Salicylic acid activates nitric oxide synthesis in Arabidopsis. J. Exp. Bot. 58, 1397-1405. doi: 10.1093/jxb/erm001

Conflict of Interest: The authors declare that the research was conducted in the absence of any commercial or financial relationships that could be construed as a potential conflict of interest.

Copyright $\odot 2020$ Nejamkin, Foresi, Mayta, Lodeyro, Del Castello, Correa-Aragunde, Carrillo and Lamattina. This is an open-access article distributed under the terms of the Creative Commons Attribution License (CC BY). The use, distribution or reproduction in other forums is permitted, provided the original author(s) and the copyright owner(s) are credited and that the original publication in this journal is cited, in accordance with accepted academic practice. No use, distribution or reproduction is permitted which does not comply with these terms. 